\title{
Summary and Abstracts of the Seventh International Kawasaki Disease Symposium
}

\author{
December 4-7, 2001, Hakone, Japan
}

\begin{abstract}
JANE W. NEWBURGER, KATHRYN A. TAUBERT, STANFORD T. SHULMAN, ANNE H. ROWLEY, MICHAEL H. GEWITZ, MASATO TAKAHASHI, BRIAN W. MCCRINDLE

Children's Hospital, Harvard University, Boston, Massachusetts [J.W.N.]; American Heart Association, Dallas, Texas [K.A.T.]; The Children's Memorial Hospital, Northwestern University Medical School, Chicago, Illinois [S.T.S., A.H.R.]; The Children's Hospital at Westchester Medical Center, NY Medical College, Valhalla, New York [M.H.G.]; Childrens Hospital Los Angeles and the University of Southern California School of Medicine [M.T.]; and The Hospital for Sick Children, University of Toronto,
\end{abstract} Toronto, Ontario, Canada [B.W.M.]

The Seventh International Kawasaki Disease Symposium, sponsored by the Japan Kawasaki Disease Research Center, American Heart Association, and Japan Heart Foundation, brought together clinicians and researchers from throughout the world to report on the state of the art related to Kawasaki disease. Despite the terrorist attack of September 11, 2001, attendance reached an all-time high in the beautiful setting of the Fuji-Hakone-Izu National Park in Japan. The Symposium format included the Hideko Ogawa, Yuki Takahashi, and Richard Rowe Memorial Lectures, round table discussions, state of the arts sessions, oral abstract presentations, and both moderated and general poster presentation sessions. We have attempted to briefly capture the highlights of the Symposium; the individual abstracts follow the summary.

\section{EPIDEMIOLOGY}

\section{Kathryn A. Taubert}

Several presentations provided epidemiologic updates on Kawasaki disease (KD). Japan, the country with the most reported cases of $\mathrm{KD}$, has conducted nationwide surveys since 1970; through the end of 2000 , they have recorded 168,394 cases. The incidence rates were higher in males and in children age 1 year or younger. For the year 2000, the incidence rate was 134.2 per 100,000 children age $<5$ year [Abstract \#004].

Other reports from Asia were presented. In Taiwan, 4,892 cases of KD occurred between 1994 and 2000, and the annual incidence rate for the year 2000 was 53.3 cases per 100,000 children $<5$ years old [Abstract \#005]. Another report from urban medical centers in four Chinese provinces over a 5 -year period reported annual incidence rates of 1.85 to 24.4 per 100,000 children age $<5$ year [Abstract \#006]. A survey of

Brian McCrindle, M.D., The Hospital for Sick Children, 555 University Avenue, Toronto, Ontario, Canada M5G 1X8; e-mail: brian.mccrindle@sickkids.ca
Hong Kong hospitals for 1994-2000 found an average annual incidence rate of 32 per 100,000 children age $<5$ year [Abstract \#007].

For North America, researchers surveyed hospitals in Ontario, Canada, from 1998-2000 and reported an annual incidence rate of 20.6 cases per 100,000 children age $<5$ year [Abstract \#008]. This was a $42 \%$ increase in incidence compared with their previous 3-year survey. Regional studies within the United States were also presented. Researchers from Childrens Hospital of Los Angeles, CA (which serves a large urban, multi-ethnic population) presented a report on their cases over the 21-year period of 1979-2000, and found an attack rate of 67.2 cases per 100,000 children age $<5$ year [Abstract \#009]. A study from the state of Georgia found an annual incidence rate of 9.8 per 100,000 children age $<5$ year for 1997-1998 [Abstract \#053].

Researchers from England examined emergency admissions for KD between 1991 and 2000 [Abstract \#177]. They found that the incidence rate per 100,000 children age $<5$ year doubled from 4.0 in $1991-1992$ to 8.1 in $1999-2000$.

\section{ETIOLOGY}

\section{Stanford T. Shulman}

The unresolved controversy regarding the etiology of KD again focused primarily upon the possibilities 1 ) that a specific conventional agent and immune response causes $\mathrm{KD}$, or 2) that a superantigen-induced immune response serves as the trigger for the disease. The former possibility was supported by the studies of Rowley et al. [Abstract \#013] who have developed synthetic oligoclonal IgA KD antibodies by transfection of oligoclonal immunoglobulin heavy and light chain genes from a fatal case of KD. Specific staining of KD tissues by synthetic antibody was demonstrated. Peters et al. [Abstract \#064] reported finding a novel cDNA sequence derived from peripheral 
blood cells of acute stage KD patients. This sequence shared low-level homology with a gene of African swine fever virus, a lymphotropic poxvirus.

Several other studies supported the superantigen hypothesis of KD. Burgner et al. [Abstract \#010] reported that a somewhat greater proportion of $\mathrm{KD}$ patients than controls had nasal colonization with Staphylococcus aureus that showed mitogenic (superantigen) activity in sterile culture supernatants and/or that contained superantigen-encoding genes. The authors suggested that any one of many different staphylococcal superantigens might be etiologically related to KD. Suzuki et al. [Abstract \#011] reported polyclonal activation of $\mathrm{V} \beta 2$ and $\mathrm{V} \beta$ 6.5- bearing $\mathrm{T}$ cells in acute $\mathrm{KD}$, as well as serum IgM antibodies to streptococcal pyrogenic exotoxin C (SPE C) that were present in lower titer in the convalescent phase compared with the acute phase of KD. Nomura et al. [Abstract \#012] measured serum levels of antibody to two streptococcal (SPE A, SPE C) and two staphylococcal (TSST-1, SEB) superantigens in 15 untreated KD patients age $<6$ months, 10 mothers of very young (age $<6$ months) KD patients, 40 normal adults, and 22 control infants age $<6$ months. The authors found lower anti-TSST-1 titers among mothers of KD babies compared with adult controls. In 6 of 8 infant-mother pairs, the infants had higher anti-TSST-1 levels than their mothers. The authors interpreted this as supporting a role for TSST-1 in acute KD.

\section{PATHOGENESIS}

\section{Anne H. Rowley}

The role of activated monocytes/macrophages, neutrophils, and lymphocytes in the pathogenesis of $\mathrm{KD}$ was explored in a number of presentations. Furukawa et al. [Abstract \#016] reported that $\mathrm{CD} 14+$ monocytes/macrophages in peripheral blood from patients with acute KD had interesting structural characteristics by electron microscopy, including abundant intracytoplasmic granules, the quantity of which correlated with disease severity. These authors also noted more NF- $\kappa$ B activation in $\mathrm{CD} 14+$ monocytes/macrophages than in $\mathrm{CD} 3+\mathrm{T}$ cells in acute KD. Terai et al. [Abstract \#017] also noted increased macrophage activation in acute KD. Macrophage infiltration of arterial tissue was noted by two groups, Brown et al. [Abstract \#019] and Jennette et al. [Abstract \#094], both of whom also noted infiltration of arterial tissue by lymphocytes, with $\mathrm{CD} 8+\mathrm{T}$ cells much more prevalent than $\mathrm{CD} 4+\mathrm{T}$ cells. Adrenomedullin, a vasodilatory protein produced by macrophages and lymphocytes, was found to be up-regulated in acute KD by Nomura et al. [Abstract \#078] and Nishida et al. [Abstract \#079]. Neutrophil elastase, secreted by activated neutrophils, was found to be elevated in acute KD by Biezeveld et al. [Abstract \#085]. Fukazawa et al. [Abstract $\# 068]$ reported an increase in Mac-1 expression on leukocytes in acute $\mathrm{KD}$, with enhanced leukocyte adhesion to endothelial cells. Matsubara et al. [Abstract \#146] reported that cytotoxic $\mathrm{T}$ lymphocyte-associated antigen 4 was up-regulated in the acute stage of KD. Chua et al. [Abstract \#021] reported that human coronary artery endothelial cells treated with TNFalpha express adhesion molecules and secrete macrophage chemotactic protein-1, proposing that this pathway allows for leukocyte infiltration into arterial tissue. Shingadia et al. [Abstract \#089] reported a decrease in the absolute numbers of B lymphocytes expressing surface and cytoplasmic IgA in acute $\mathrm{KD}$, speculating that IgA B lymphocytes move selectively from the peripheral circulation into the target tissues.

Matrix metalloproteinases (MMP) were the subject of a number of presentations. Gavin et al. [Abstract \#069] reported increased MMP-9 detection in coronary aneurysms from acute $\mathrm{KD}$ patients when compared with controls. Sakata et al. [Abstract \#030] reported a potential mechanism of regulation of synthesis of MMP-9 by endothelial cells in KD by human hepatocyte growth factor, secreted by fibroblasts stimulated by other cytokines. Chua et al. [Abstract \#071] reported that elevated serum levels of MMP-9 and tissue inhibitor of metalloproteinase-1 in acute KD might be useful in developing a much-needed diagnostic test; similar elevated levels were reported by Sekiguchi et al. [Abstract \#072].

Changes in peripheral leukocyte apoptosis in acute KD were the focus of several presentations. Abe et al. [Abstract \#020] reported that apoptosis inhibitory proteins were up-regulated in the acute phase of KD. Yi et al. [Abstract \#073] also reported inhibition of lymphocyte apoptosis in acute KD, and Tsujimoto et al. [Abstract \#076] found that neutrophil apoptosis was also inhibited in the acute phase.

\section{GUIDELINES FOR KAWASAKI DISEASE}

\section{Jane W. Newburger}

Several presentations in a Round Table discussion focused on the need for revision of guidelines for KD. The Kawasaki Disease Research Committee (Ayusawa et al. for the Subcommittee for the Revision of Diagnostic Guidelines [Chief: $\mathrm{T}$ Sonobe]) presented their proposal to revise the guidelines. Because approximately $30 \%$ of children in the 16th Japanese nationwide surveillance were treated on or before the 4th day of illness, new guidelines are likely to require only four febrile days. In addition, plans for guideline revision include increased emphasis on atypical or incomplete cases of $\mathrm{KD}$, in whom coronary artery lesions have an incidence similar to that in classic KD [Abstract \#032]. Burns et al. [Abstract \#038] presented the results of a survey of general pediatricians in San Diego County and U.S. members of the Pediatric Infectious Disease Society. Half of general pediatricians and $90 \%$ of pediatric infectious diseases specialists reported having made the diagnosis of KD in febrile patients who did not fulfill four of the five principal clinical criteria. Both groups were insufficiently aware that $\mathrm{KD}$ could occur in infants younger than age 6 months or older than 8 years. Bastian et al. [Abstract \#034] reported a sensitivity of the current Center for Disease Control case definition of only $81 \%$ for detecting children with coronary abnormalities; thus, reliance on this case definition as the sole criteria for treatment results in physician failure to detect children at risk for coronary abnormalities. These authors stressed that specificity of the treatment criteria is less important than high sensitivity, in that the treatment is effective and relatively safe. Some Round Table participants believed that incorporation of laboratory measures and important nonprinci- 
pal symptoms would likely improve sensitivity without undue reduction in specificity. Future challenges include ensuring consistency in the epidemiologic definition of the disease so that years of meticulous nationwide surveys in Japan have continuity with present surveillance.

\section{TREATMENT IN THE ACUTE PHASE}

\section{Jane W. Newburger}

Comparisons of i.v. gamma globulin (IVIG) products and of different dose regimens were the subject of several presentations. A retrospective study by Iwasa et al. [Abstract \#131] suggested that IVIG prepared using propylene glycol was associated with better outcomes than sulfonated IVIG. Similarly, Muta et al. [Abstract \#138] retrospectively compared three different products: the incidence of coronary artery complications was $10 \%$ with a sulfonated product, $8.9 \%$ with a pH4-treated product, and $2.8 \%$ with a polyethylene glycoltreated product. Because IVIG is expensive in Japan, several studies examined the use of a flexible $1 \mathrm{~g} / \mathrm{kg}$ dosage regimen, allowing retreatment if fever and severe inflammation were persistent. Ogino et al. [Abstract \#130] reported a higher likelihood of requirement for retreatment in patients receiving a lower initial dose of IVIG. Ozawa et al. [Abstract \#137] performed a randomized study of single infusion of IVIG $2 \mathrm{~g} / \mathrm{kg}$ versus $1 \mathrm{~g} / \mathrm{kg}$ as initial therapy for KD; none of 36 children in the higher-dose group developed coronary abnormalities, whereas 2 of 41 children treated with the lower dose regimen had such abnormalities. Some investigators continue to use the Harada score to determine the need for IVIG treatment [Abstract \#140]. Sung et al. [Abstract \#136] performed a metaanalysis demonstrating that IVIG in a $2 \mathrm{~g} / \mathrm{kg}$ dosage regimen was more effective in preventing coronary abnormalities than a flexible dosage regimen of $1 \mathrm{~g} / \mathrm{kg}$.

Several groups focused on the efficacy of early treatment of KD. In a case-control study, Fong et al. [Abstract \#127] found that IVIG treatment given earlier than day 5 after the onset of illness resulted in a higher likelihood of IVIG retreatment but a similar incidence of coronary abnormalities. Similarly, Ogino et al. [Abstract \#130] reported that children treated on the 2nd to 4th day of illness were significantly more likely to require additional IVIG. In contrast, Sugahara et al. [Abstract \#127] found that children treated before day 5 after the onset of illness compared with those treated later, had a shortened total duration of fever, again with similar coronary outcomes. Finally, Tse et al. [Abstract \#128] found that early treatment, before day 6 after the onset of illness, compared with treatment on days 7-10, resulted in less coronary ectasia 1 year later, but was not associated with fever duration, length of hospitalization, or development of coronary aneurysms.

Several studies focused specifically on retreatment with IVIG. Among patients receiving additional IVIG treatment, Miura et al. [Abstract \#129] reported that patients who were retreated within $10 \mathrm{~d}$ of the onset of fever and in whom the fever had resolved within 2 days had lower risk of coronary artery abnormalities. Durongpisitkul et al. [Abstract \#132] retrospectively reviewed all children admitted with KD to determine the occurrence of and variables associated with initial IVIG treatment failure. They found that $11.5 \%$ of children treated with a single dose of $2 \mathrm{~g} / \mathrm{kg}$ of IVIG were subsequently retreated with IVIG, and that children with low $\mathrm{Hb}$, high white blood count, and high neutrophil count were more likely to receive retreatment. Similar to findings in previously published studies, these factors were also predictive of coronary artery abnormalities. Sano et al. [Abstract \#134] presented a prediction model for resistance to IVIG using baseline laboratory data. In logistic regression, those with higher C-reactive protein, bilirubin, and AST levels were found to be more likely to be IVIG resistant. However, this model was not tested prospectively. Consistent with published literature, various studies confirmed that IVIG resistance (i.e. persistent fever after IVIG) was associated with development of coronary artery abnormalities.

Several presentations addressed steroid treatment for KD. Sundel et al. [Abstract \#037] conducted a prospective randomized open-label clinical trial to determine whether the addition of pulsed i.v. methylprednisolone (IVMP), 30mg/kg, to standard therapy of IVIG $(2 \mathrm{~g} / \mathrm{kg})$ and aspirin might improve outcomes. These investigators reported that treatment with IVMP plus IVIG resulted in faster resolution of fever, more rapid improvement in serum markers of inflammation, and shorter length of hospitalization. Adverse effects attributable to IVMP were rare. The treatment groups were similar in coronary artery dimensions, but the study was underpowered to detect a difference. Kang et al. [Abstract \#038] conducted a retrospective review of the clinical outcome of high-risk patients with KD treated with various treatment regimens, with or without steroids. Coronary outcomes were similar in treatment groups, but those treated with prednisone had decreased fever duration. Similarly, Jibiki et al. [Abstract \#148] demonstrated shortened fever course when combining dexamethasone with IVIG, but no change in coronary artery outcomes. A retrospective review by Shinohara et al. [Abstract \#142] suggested a role for oral prednisolone therapy. Larger prospective randomized trials will be necessary before conclusions can be reached about the effect of steroid therapy on coronary artery outcomes in $\mathrm{KD}$.

Case series of patients treated with other modalities were also presented. Takahashi et al. [Abstract \#040] presented a series of eight patients with large coronary artery aneurysms treated with abciximab. Although abciximab was well tolerated, it did not produce dramatic regression of coronary artery aneurysms as had been previously suggested. Retrospective case series concerning ulinastatin, a urinary trypsin inhibitor that inhibits neutrophil elastase activity, were inconclusive (positive effect reported by Yoshida et al. [Abstract \#144]; negative effect reported by Fuse et al. [Abstract \#143]). Miyamae et al. [Abstract \#147] treated 50 high-risk patients with plasma exchange once daily for each of three successive days, with apparent benefit.

Symposium participants recognized remaining challenges in the therapy of KD. Because the incidence of coronary artery abnormalities is very low in patients treated with high-dose IVIG in the acute phase, multi-center randomized trials are needed to accrue a large enough sample size to achieve sufficient statistical power. 


\section{STRESS TESTING AND IMAGING}

\section{Michael H. Gewitz}

Stress testing. Dynamic cardiac performance testing was discussed in the context of the longitudinal assessment of the child with KD. Several presentations focused exclusively on this topic, whereas others included stress testing, either exercise or pharmacologic, as one of a variety of measures of long-term outcome with regard to myocardial ischemia and development of coronary artery stenosis. The largest study [Abstract \#154] involved 70 patients undergoing dipyridamole stress Tc-99m SPECT studies, suggesting the method to be a sensitive noninvasive indicator of ischemia secondary to coronary artery stenosis. These results were confirmed with coronary angiography. Dipyridamole stress imaging with myocardial contrast echo was also presented as a potential means to detect myocardial perfusion deficits, avoiding use of radionuclides. Only 20 patients were included in the study presented [Abstract \#155], but the technique may be promising for serial assessment. Exercise scintigraphy was also used to calculate fractional myocardial flow reserve and coronary flow reserve as referenced to papaverine challenge [Abstract \#022], with results suggesting that these indices may detect myocardial ischemia both before and after catheter and/or surgical interventions. Other methods of assessment for coronary artery changes, including ultrafast or electron beam computed tomography (CT), and magnetic resonance (MR) angiography, were discussed and may have potential, but the numbers of patients in each report were too few for definitive assessment. An interesting survey of U.S. pediatric cardiologists suggested that stress evaluations, either with echo or perfusion scanning, are used in follow-up programs by a large number of clinicians even for patients with relatively low risk for sequelae, as defined by the 1994 American Heart Association Kawasaki disease guidelines [Abstract \#042].

Imaging. Gadolinium-enhanced MR angiography may be useful for thrombus detection in giant coronary artery aneurysms, but only three patients were studied with this technique [Abstract \#024]. Other promising methods for static assessment of myocardial damage secondary to coronary artery abnormalities, which were presented, included ultrasonic tissue characterization, which appears to correlate with VEGF kinetics [Abstract \#025] and perhaps intravascular ultrasound [Abstract \#043]. These methods require more substantiation and corroborative studies. Much attention was focused on standard 2D echocardiography/Doppler and its potential inclusion as a diagnostic criterion for KD [Abstract \#114]. In particular, changes on $2 \mathrm{D}$ echocardiography/Doppler involving valvular flow profiles, myocardial or pericardial changes, as well as coronary artery findings, may be useful markers for patients in whom some of the classical clinical features may be missing, so called "incomplete KD." Results of a San Diego area survey [Abstract \#033] suggest clinicians may already be using findings from echocardiography to aid in the diagnosis of $\mathrm{KD}$. The echocardiographic criteria for coronary artery involvement were discussed extensively with a consensus beginning to emerge from both sides of the Pacific ([Abstract \#112],[Abstract \#108], and others) that measurement of coronary artery dimensions should be compared with standardized reference values, as opposed to using the Japanese Ministry of Health guidelines. Finally, Takahashi et al. [Abstract \#095] reported that "perivascular brightness" seen in the coronary arterial wall by echocardiography early in the acute phase of the disease might be a predictor of long-term coronary artery abnormality. This marker may be useful even when initial lumen diameter is not increased, but more data are needed to verify this observation.

\section{CORONARY ARTERY INTERVENTIONS}

\section{Masato Takahashi}

Surgical revascularization. There is no question that Japanese colleagues are ahead of North Americans or Europeans in terms of their experience with coronary artery revascularization in KD patients, although accurate total nationwide statistics for success rate or mortality/morbidity rates have not been made available. Dr. Kitamura at National Cardiovascular Center, Osaka, Japan, has the largest experience, with more than 100 cases thus far, targeting severely stenotic or occluded coronary arteries [Abstract \#026]. He has previously reported much higher patency of arterial grafts compared with venous grafts, and also noted that the arterial grafts (mainly internal thoracic artery grafts) showed excellent potential for growth in both caliber and length, keeping up with normal somatic growth of the children. Nakano et al. from Nippon Medical School, Tokyo, reported a series of 13 cases with arterial grafts, targeting not only already obstructed native vessels but also placing grafts beyond some large coronary aneurysms with no associated obstructions for prevention of future ischemic events [Abstract \#031]. No mortality or major morbidity was reported in this series. However, many of the large aneurysms tended to show "complete degradation" following surgery. A lively discussion among the panelists indicated no universal agreement about necessity of such preemptive surgery.

Catheter intervention. Dr. Akagi, Kurume University, reported on nationwide statistics on catheter intervention [Abstract \#028]. A total of 88 patients with 100 lesions were treated with catheter intervention from 1990 to 2001. Pediatric and adult interventional cardiologists collaborated in most of these cases. The patients were followed for at least 3 months. Type of procedure included balloon angioplasty (PTCA) (21 procedures), rotational ablation (PTCRA) (68 procedures) and stent placement (11 procedures). Some patients received stent placement combined with either PTCA or PTCRA. Patients who had PTCRA were generally older (mean age of 15 years), with a much longer interval from the acute illness compared with those who had PTCA. Although all three procedures were initially successful in over $80 \%$ of cases, restenosis and neoaneurysm formation occurred to varying degrees. Restenosis was more prevalent in those who had PTCA (33\% compared with 21\% [PTCRA] and 9\% [stent]). Neoaneurysms were noted in $6 \%$ to $7 \%$ among both PTCA and PTCRA cases. It was noted that in post-Kawasaki coronary artery stenosis, high balloon pressures of 10 to $15 \mathrm{~atm}$ were required to effect dilation, and this may have contributed to neoaneurysms. Small 
numbers of patients who had PTCA or PTCRA underwent repeat catheter interventions, but none of the patients who had stent placement needed reintervention. Four of 21 patients who received PTCA were eventually referred for surgical revascularization.

\section{LONG-TERM OUTCOMES}

\section{Brian W. McCrindle}

Several key aspects of long-term outcomes after KD remain of ongoing concern. Data regarding the mechanisms and sequelae of persistence or regression of coronary artery lesions were presented. Pyle et al. [Abstract \#108] showed that, in an analysis of serial measurements of normalized coronary artery segments, dimensions regressed early in ectatic segments, with subsequent dimensions significantly related to the initial dimensions and the dimensions of other segments. In addition, while giant aneurysms were previously felt to not regress, Miller et al. [Abstract \#164] noted evidence of regression with a low incidence of ischemia, regardless of the use of long-term anticoagulation. Ishii et al. [Abstract \#043] used intravascular ultrasound to show that patients with persistent and regressed coronary artery aneurysms had abnormal vascular wall morphology, with increased intima-media thickening and variable degrees of calcification and impaired vascular elasticity, whereas uninvolved segments were normal.

Controversy continues as to whether $\mathrm{KD}$ has long-term effects, both in coronary arteries and more generally in systemic arteries that would indicate an increased risk of premature atherosclerosis. Noninvasive assessments of endothelial dysfunction, an early marker for atherosclerosis, have shown conflicting results. McCrindle et al. [Abstract \#163] showed in a long-term case-control study that KD patients were more likely to have impairments of the fibrinolytic system, a marker for endothelial dysfunction, and this was unrelated to the degree of coronary artery involvement. In contrast, no differences in brachial artery reactivity, carotid intima-media thickness, or any other cardiovascular risk factor were noted by McCrindle et al. [Abstract \#167] in a cross-sectional casecontrol study, although there was some evidence to suggest impaired blood pressure regulation on 24-hour ambulatory monitoring in cases. Mitani et al. [Abstract \#166] noted endothelial dysfunction in both involved and uninvolved coronary artery segments, but not in femoral arteries. On a pathologic study, Takahashi et al. [Abstract \#168] noted long-term coronary arterial scarring in five of six patients without echocardiographic evidence of luminal changes in the acute phase. Increased coronary artery calcification, as noted by ultrafast computed tomography by Dadlani et al. [Abstract \#161] may indicate an increased predisposition to atherosclerosis in involved coronary artery segments. Likewise, subtle long-term cardiologic findings were noted by Watanabe and Oota $[\mathrm{Ab}-$ stract \#172] in patients without initial echocardiographic abnormalities, particularly in those presenting with $\mathrm{KD}$ at very young ages. Based on the studies presented, it appears that the risk of long-term vascular abnormalities appears to be limited to the coronary arteries and may occur even in the absence of echocardiographic evidence of initial involvement.

The long-term outlook for patients with coronary aneurysms remains guarded, as was reviewed by Dr. Kato. In the fifth look at long-term outcomes of 6,576 persons after KD, Nakamura et al. [Abstract \#044] showed that the mortality rate for patients without cardiac sequelae and female patients with sequelae did not differ from the normal population, whereas males with sequelae had a mortality rate 2.4 times higher than expected. However, Wong and McCrindle [Abstract \#174] showed that long-term health status is normal after $\mathrm{KD}$ and not related to disease characteristics, although a trend toward lower health status with increasing age may indicate problems with adolescent adjustment. 